\title{
Hyperbolic reformulation approach to enable efficient simulation of groundwater flow and reactive transport
}

\author{
Ilhan Özgen-Xian ${ }^{1, *}$, Adrián Navas-Montilla², Dipankar \\ Dwivedi $^{1}$, and Sergi Molins ${ }^{1}$ \\ ${ }^{1}$ EESA, Lawrence Berkeley National Laboratory, CA, USA \\ ${ }^{2}$ Centro Universitario de la Defensa, Zaragoza, Spain \\ *Corresponding author at Lawrence Berkeley National Laboratory, 1 Cyclotron \\ Road, M/S 74R316C, Berkeley, CA947230, USA. E-mail: iozgen@lbl.gov
}

\section{Preprint}

Final version appears in Environmental Engineering Science

Special Issue on Addressing Society's Water and Energy Challenges with Reactive Transport Modeling

doi: $10.1089 /$ ees.2020.0363

\begin{abstract}
We apply Cattaneo's relaxation approach to the one-dimensional coupled Boussinesq groundwater flow and advection-diffusion-reaction equations, commonly used in engineering applications to simulate contaminant transport in the subsurface. The diffusion-type governing equations are reformulated as a hyperbolic system, augmented by an equation that can be interpreted as a momentum balance. The hyperbolization enables an efficient unified computation of the primary variable and its gradients, for example piezometric head and unit discharge in the Boussinesq equation. An augmented Roe scheme is used to solve the hyperbolic system. The hyperbolized system of equations is studied in a set of steady state and transient test cases with idealized geometry. These test cases confirm the equivalence of the hyperbolic system to its original formulation. The larger time step size of the hyperbolic equation is verified theoretically by means of a stability analysis and numerically in the test cases. Finally, a reach-scale application of flow and transport across a river meander is considered. This application case shows that the performance of the hyperbolic relaxation approach holds for more realistic groundwater flow and transport problems, relevant to water resources management.
\end{abstract}

Key words: groundwater flow; advection-diffusion-reaction equation, hyperbolic relaxation system; augmented Roe scheme 


\section{Contents}

1 Introduction 2

2 Governing equations 4

2.1 Reactive transport in groundwater . . . . . . . . . . . . . 4

2.2 Hyperbolic groundwater flow equation . . . . . . . . . . . 5

2.3 Hyperbolic advection-diffusion-reaction equation . . . . . . 6

$\begin{array}{lll}3 & \text { Numerical scheme } & \mathbf{7}\end{array}$

3.1 Augmented Roe scheme . . . . . . . . . . . . . . . . . 7

3.2 Source term discretization . . . . . . . . . . . . . . . 8

3.3 Boundary conditions $\ldots \ldots \ldots \ldots \ldots$

$\begin{array}{lll}3.4 & \text { Comparison between parabolic and hyperbolic time step size } & 9\end{array}$

$\begin{array}{llr}4 \text { Model verification } & 10\end{array}$

4.1 Subsurface flow test cases . . . . . . . . . . . . . . . 10

$4.1 .1 \quad$ Steady state test cases . . . . . . . . . . . . . 10

4.1 .2 Transient test case . . . . . . . . . . . . . . . . 11

4.2 Reactive transport test cases . . . . . . . . . . . . . . . 12

$4.2 .1 \quad$ Steady state test cases . . . . . . . . . . . . . . 12

4.2 .2 Transient test cases . . . . . . . . . . . . . . . . . 12

5 Modeling intrameander hyporheic flow and transport 14

$\begin{array}{lll}6 & \text { Conclusions } & 19\end{array}$

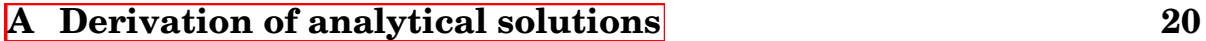

A.1 Subsurface flow . . . . . . . . . . . . . . . 20

A.1.1 Steady state solutions . . . . . . . . . . . 20

A.2 Reactive transport . . . . . . . . . . . . . . . . 20

A.2.1 Transient solutions . . . . . . . . . . . . . . . 20

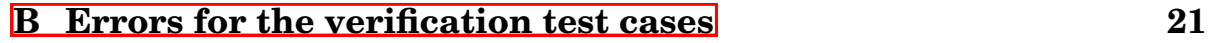

\section{Introduction}

Reactive transport models (RTMs) are widely used to investigate coupled physical and biogeochemical processes in natural and engineered systems [28]. Mechanistic insights gained with RTMs have broad relevance for water, energy, and the environment with applicability from the critical zone to the deep subsurface [18, 6]. RTM simulations are underpinned by the numerical solution of a set of mixed differential and algebraic equations that often have significant computational costs [28]. 
The differential equations that describe groundwater flow and solute transport that are part of RTMs usually include parabolic terms. Examples of these type of equations are the Boussinesq groundwater flow equation (BE) and the advection-diffusion-reaction equation (ADR). The BE is a nonlinear parabolic partial differential equation (PDE) and the ADR is a mixed-type parabolic-hyperbolic PDE. The parabolic nature of these equations poses two challenges. Firstly, the time step size of the BE is constrained by a parabolic constraint of $O\left(h^{2}\right)$ [30]. Secondly, the unit discharge in the BE is not a primary conserved variable, but instead is computed from the gradient of the piezometric head. Thus, the piezometric head and the unit discharge are not necessarily computed with uniform accuracy [22]. The same challenges apply to the parabolic part of the ADR. Reformulating the equations in hyperbolic form makes it possible to overcome these issues which hamper efficient simulation [22, 30]. In this work, we apply a relaxation approach to convert the $\mathrm{BE}$ and $\mathrm{ADR}$ into a first order hyperbolic system with the objective of enabling efficient simulations. The BE and single-component ADR are used here as examples of the general groundwater flow and reactive transport equations but also because they are commonly used in water resources management [2]. They also make it possible to simplify simulations of unconfined systems dominated by horizontal processes when the Dupuit assumptions hold [1]. In order to model contaminant spreading-or the transport of any arbitrary chemical species-in the subsurface, the BE can be coupled with the advectiondiffusion-reaction equation (ADR).

The relaxation approach employed in this work was developed in [22] to obtain steady state solutions for a diffusion equation using a residual distribution approach, and is extended to the Navier-Stokes equations in [24]. Transient solutions have been proposed for advection-diffusion equations in [19], and more recently for the heat equation in [11]. However, the relaxation approach has not yet been applied to groundwater flow and reactive transport problems. The specific relaxation approach used in these publications is Cattaneo's relaxation approach [3]-see [30] for a discussion of the advantage of this particular relaxation approach over other existing ones. In the literature, Cattaneo's relaxation approach is considered as a solely numerical treatment that hyperbolizes diffusion-type equations. More generally, for example in [14, 13], relaxation approaches enforce the hydrodynamic limit to kinetic models of the Boltzmann equation through diffusive rescaling. However, this interpretation is not valid for Cattaneo's relaxation. Following the discussion in [16], we consider the relaxation system to be an equilibrium system that is subject to perturbations. The equilibrium state of this system is the original diffusion-type equation. The hyperbolization can then be thought of as a (small) perturbation around this equilibrium state, that allows to solve the system more efficiently. The system relaxes to its equilibrium state, that is converges toward the diffusiontype equation, fast enough, such that the perturbation has negligible effect on the model solution. 
When applied to the $\mathrm{BE}$ and $\mathrm{ADR}$, the resulting first order hyperbolic equations (hereinafter referred to as $\mathrm{hBE}$ and $\mathrm{hADR}$, respectively) may be written with the unit discharge as a primary variable. Thus, it may be computed simultaneously with the piezometric head or the species concentration and with uniform accuracy. The time step size limitation is formally relaxed to $O(h)$, although, as shown in [23], the parabolic constraint of $O\left(h^{2}\right)$ is implicitly contained in the Courant-Friedrichs-Lewy condition of the hyperbolic system [7].

In what follows, we derive the $\mathrm{hBE}$ and $\mathrm{hADR}$ and discuss some of their properties. We present an augmented Roe scheme to solve these systems. We carry out transient and steady state benchmarks that verify that the hyperbolized equations converge to their parabolic counterparts. We follow by bringing to bear the new approach on the simulation of hyporheic zone flow and reactive transport across a river meander of the East River, Colorado, USA [9]. The hyporheic zone is a benthic dynamic ecotone where nutrientrich groundwater and oxic surface water interact and consequently form a redox gradient along the flow path. In this application, the flow and transport across the meander region is driven by the difference in river heads at both sides of the meander. Flow is mostly horizontal and biogeochemical processes in the meander region result in a horizontal redox zonation. From these simulations, we conclude that the first order hyperbolic system is competitive for use with numerical schemes with explicit time integration.

\section{Governing equations}

\subsection{Reactive transport in groundwater}

The Boussinesq groundwater flow equation (BE) describes unconfined, saturated flow in nearly horizontal aquifers. Coupling it with an advectiondiffusion-reaction equation (ADR) gives

$$
\left.\begin{array}{l}
\partial_{t} m_{f}-\partial_{x}\left(h v_{f}\right)=0 \\
\partial_{t} m_{c}-\partial_{x}\left(D m_{f} \partial_{x} c\right)-\partial_{x}\left(h v_{f} c\right)=-R
\end{array}\right\}
$$

Here, $t$ is time, $m_{f}=S_{y} h$ is the mass of the fluid per unit width, where $S_{y}$ is the specific yield of the soil and $h$ is the height of the aquifer; $x$ is the coordinate axis; $v_{f}$ is the Darcy velocity, calculated as

$$
v_{f}=K(x) \partial_{x} \eta,
$$

with $\eta$ being the piezometric head and $K$ being the hydraulic conductivity of the soil. The aquifer height relates to the piezometric head as

$$
h=\eta-z,
$$




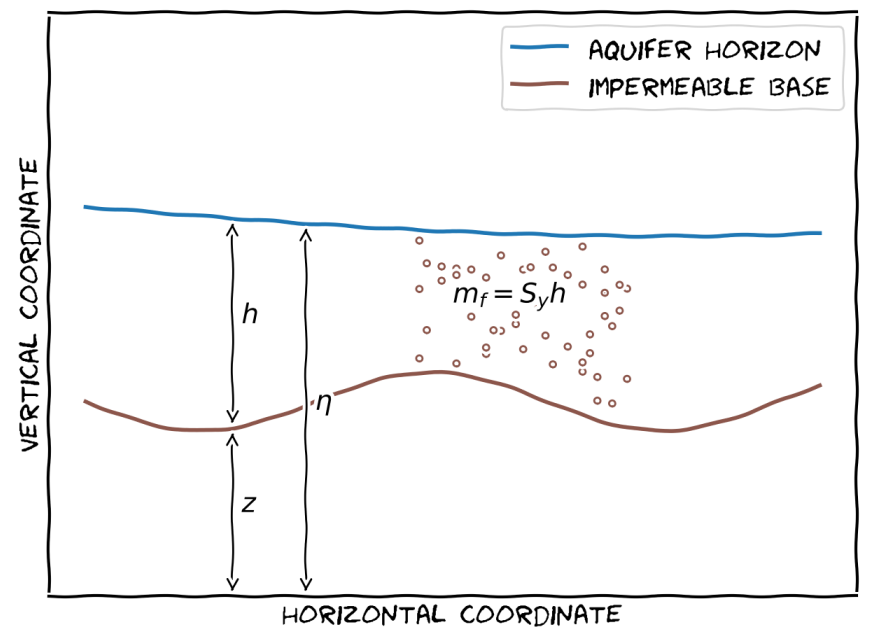

Figure 1: Definition sketch for the variables used in the BE and ADR.

where $z$ is the impermeable base datum. A definition sketch for these variables is shown in Fig. 11. $m_{c}=S_{y} h c$ is the mass of the species per unit width, with $c$ being the concentration of this species; $D$ is the dispersion coefficient; and $R$ is a (nonlinear) function that describes a reaction process. Eq. (1) describes the conservation of mass for fluid and a single chemical species.

\subsection{Hyperbolic groundwater flow equation}

Using Cattaneo's relaxation approach [3], the first equation in Eq. (1) can be written as a first order hyperbolic system as

$$
\left.\begin{array}{l}
\partial_{t} m_{f}-\partial_{x} q_{f}=0, \\
\partial_{t} q_{f}-\frac{K m_{f}}{S_{y} \tau} \partial_{x} m_{f}=-\frac{q_{f}}{\tau}+\frac{K m_{f}}{S_{y} \tau}\left(\partial_{x} z-\frac{m_{f}}{S_{y}^{2}} \partial_{x} S_{y}\right),
\end{array}\right\}
$$

where we have split $\eta$ in the components $h$ and $z$ and used the chain rule to split the product of $m_{f}$ and $S_{y} . \tau$ is the relaxation parameter, and $q_{f}$ is the unit discharge of the fluid that converges to $K h \partial_{x} \eta$ as $\tau$ goes to zero. Thus, we may interpret the hyperbolization as a perturbation around an equilibrium state that corresponds to the original diffusion-type equation. Here, $\tau$ determines at which rate the hyperbolic system in Eq. (4) relaxes towards its equilibrium state, that is to say the original diffusion-type equation. The smaller $\tau$ is, the faster the system converges. 
Sys. (4) can be written in vector form as

$$
\begin{aligned}
& \partial_{t} Q+A \partial_{x} Q=S, \\
& Q=\left[\begin{array}{c}
m_{f} \\
q_{f}
\end{array}\right], A=\left[\begin{array}{cc}
0 & -1 \\
-K m_{f} S_{y}^{-2} \tau^{-1} & 0
\end{array}\right], \\
& S=\left[\begin{array}{c}
0 \\
\tau^{-1}\left(-q_{f}+K m_{f} S_{y}^{-1}\left(\partial_{x} z-m_{f} S_{y}^{-2} \partial_{x} S_{y}\right)\right)
\end{array}\right] .
\end{aligned}
$$

The eigenpairs of the coefficient matrix $A$ are

$$
\left.\begin{array}{l}
\lambda^{1,2}=\mp \sqrt{\frac{K m_{f}}{S_{y}^{2} \tau}}, \\
R^{1,2}=\left[\begin{array}{c}
\left(\lambda^{2,1}\right)^{-1} \\
1
\end{array}\right],
\end{array}\right\}
$$

where $\lambda^{p}$ is the $p$-th eigenvalue, and $R^{p}$ is the associated $p$-th right eigenvector. Since all the eigenvalues are real and distinct, and the eigenvectors are linearly independent, Sys. (4) is strictly hyperbolic for all nonzero values of the relaxation parameter $\tau$. The inverse of the column matrix of the right eigenvectors is

$$
\underline{\underline{R}}_{b e}^{-1}=\frac{1}{2}\left[\begin{array}{cc}
\lambda^{2} & 1 \\
\lambda^{1} & 1
\end{array}\right] .
$$

\subsection{Hyperbolic advection-diffusion-reaction equation}

Application of Cattaneo's relaxation approach to the ADR has been reported in [30], and a hyperbolic pollutant transport equation is coupled to shallow flow equations in [32]. Similar to these references, the second equation in Eq. (1) can be transformed as

$$
\left.\begin{array}{l}
\partial_{t} m_{c}-\partial_{x}\left(D m_{f} \psi_{c}-q_{f} \frac{m_{c}}{m_{f}}\right)=-R, \\
\partial_{t} \psi_{c}-\partial_{x} \frac{1}{\tau} \frac{m_{c}}{m_{f}}=-\frac{\psi_{c}}{\tau} .
\end{array}\right\}
$$

As $\tau$ goes to zero, $\psi_{c}$ converges to $\partial_{x} c$. Sys. (8) can be written in vector form as

$$
\left.\begin{array}{l}
\partial_{t} Q+\partial_{x} F(Q)=S, \\
Q=\left[\begin{array}{c}
m_{c} \\
\psi_{c}
\end{array}\right], F=\left[\begin{array}{c}
-D m_{f} \psi_{c}+q_{f} m_{c} m_{f}^{-1} \\
-m_{c} m_{f}^{-1} \tau^{-1}
\end{array}\right], \\
S=\left[\begin{array}{c}
-R \\
-\psi \tau^{-1}
\end{array}\right] .
\end{array}\right\}
$$

The Jacobian matrix of Sys. $8-J=\partial_{Q} F-$ is computed as

$$
J=\left[\begin{array}{cc}
q_{f} m_{f}^{-1} & -D m_{f} \\
-m_{f}^{-1} \tau^{-1} & 0
\end{array}\right] .
$$


The eigenpairs of $J$ are computed as

$$
\left.\begin{array}{rl}
\lambda^{3,4} & =\frac{v_{a}}{2} \mp \sqrt{\left(\frac{v_{a}}{2}\right)^{2}+\frac{D}{\tau}}, \quad v_{a}=q_{f} m_{f}^{-1}, \\
R^{3,4} & =\left[\begin{array}{c}
-\tau m_{f} \lambda^{3,4} \\
1
\end{array}\right] .
\end{array}\right\}
$$

$D$ is always positive, and thus, all eigenvalues are real and distinct. The eigenvectors are linearly independent. Hence, Sys. (8) is hyperbolic for all nonzero values of $\tau$. The inverse of the matrix of right eigenvectors is

$$
\underline{\underline{R}}_{a d r}^{-1}=\frac{1}{\tau m_{f}\left(\lambda^{3}-\lambda^{4}\right)}\left[\begin{array}{cc}
-1 & -\tau m_{f} \lambda^{4} \\
1 & \tau m_{f} \lambda^{3}
\end{array}\right] .
$$

\section{Numerical scheme}

\subsection{Augmented Roe scheme}

The governing equations are solved sequentially, using the augmented solver approach [10, 20] in fluctuation form. The cell average $Q_{i}$ in cell $i$ at time level $n$ is updated as

$$
Q_{i}^{n+1}=Q_{i}^{n}-\frac{\Delta t}{\Delta x}\left(\mathcal{A}^{+} \Delta Q_{i-1 / 2}^{n}+\mathcal{A}^{-} \Delta Q_{i+1 / 2}^{n}\right),
$$

where $\mathcal{A}^{ \pm} \Delta Q_{i \mp 1 / 2}$ are the fluctuations, measuring the net effect all ingoing waves. First, Sys. (4) is solved to provide the values of $m_{f}$ and $q_{f}$, which are then used to solve Sys. (8).

Sys. (4) is formally a nonconservative system. A difficulty in finding weak solutions to nonconservative systems is that the nonconservative product $A \partial_{x} Q$ is not well defined across discontinuities, even in a distribution sense [25]. However, if the nonconservative product is integrated along a Lipschitz continuous path $\Psi$ in phase space, it can be interpreted as a Borel measure [8]. Then, the fluctuations can be computed as

$$
\mathcal{A} \Delta Q_{i+1 / 2}=\int_{0}^{1} A(\Psi(\sigma)) \partial_{\sigma} \Psi(\sigma) d \sigma,
$$

and split into in- and outgoing fluctuations $\mathcal{A}^{ \pm} \Delta Q_{i+1 / 2}$ [17]. A common path chosen in the literature is the linear path

$$
\Psi\left(\sigma, Q_{i}, Q_{i+1}\right)=Q_{i}+\sigma\left(Q_{i+1}-Q_{i}\right),
$$

which we use in this work to construct a Roe matrix as

$$
\left\{\begin{array}{l}
\hat{A}_{i+1 / 2}=\int_{0}^{1} A\left(\Psi\left(\sigma, Q_{i}, Q_{i+1}\right)\right) d \sigma=\left[\begin{array}{cc}
0 & -1 / \hat{S}_{y} \\
-0.5 \hat{K}\left(h_{i}+h_{i+1}\right) / \tau & 0
\end{array}\right], \\
\hat{S}_{y}=0.5\left(S_{y, i}+S_{y, i+1}\right), \hat{K}=0.5\left(K_{i}+K_{i+1}\right) .
\end{array}\right.
$$


The definition of $\hat{S}_{y}$ and $\hat{K}$ is our choice. Using other averaging methods, for example, the harmonic average, does not affect the conservation property of the numerical scheme-see [25, 31] for a more detailed exposition of Roe solvers for nonconservative systems.

In the classical wave propagation approach the jump in the primary variables is split into waves [16]; the augmented solver approach extends the definition of these waves to incorporate the source terms as

$$
\left\{\begin{array}{l}
\mathcal{A}^{ \pm} \Delta Q_{i+1 / 2}=\sum_{p=1}^{m}\left(s^{p}\right)_{i+1 / 2}^{ \pm} \mathcal{Z}_{i+1 / 2}^{p}, \\
\mathcal{Z}_{i+1 / 2}^{p}=\left(\alpha_{i+1 / 2}^{p}+\frac{\beta_{i+1 / 2}^{p}}{s_{i+1 / 2}^{p}}\right) R^{p} .
\end{array}\right.
$$

$s^{p}$ is the wave speed of the $p$ th wave, $\alpha^{p}$ is the wave strength of the $p$ th wave, and $\beta^{p}$ is the source strength of the $p$ th wave [20]. These values are computed using the eigenpairs $\left\{\hat{\lambda}_{i+1 / 2}^{p}, \hat{R}_{i+1 / 2}^{p}\right\}$ of $\hat{A}_{i+1 / 2}$ from Eq. 16. Hereinafter, linearized values are denoted with a hat. We choose $s_{i+1 / 2}^{p}$ as

$$
s_{i+1 / 2}^{p}=\hat{\lambda}_{i+1 / 2}^{p} .
$$

$\alpha_{i+1 / 2}^{p}$ is calculated as the $p$ th component of the vector

$$
\underline{\underline{\alpha}}_{i+1 / 2}=\hat{R}_{i+1 / 2}^{-1} \delta Q_{i+1 / 2},
$$

where $\hat{R}^{-1}$ is the inverse of the right eigenvector matrix, and $\delta Q_{i+1 / 2}$ is the difference of cell averages across the cell interface $i+1 / 2 . \beta_{i+1 / 2}^{p}$ is computed as the $p$ th component of the vector

$$
\underline{\beta}_{i+1 / 2}=\hat{R}_{i+1 / 2}^{-1} \bar{S}_{i+1 / 2},
$$

with $\bar{S}$ being a numerical integration of the source term $S$ in Eq. (5).

The numerical solution of Sys. (8) follows the same methodology. The major difference is that Sys. (8) is formally conservative. Thus, the Jacobian matrix can be linearized in the sense of Roe [26] using the classical Rankine-Hugoniot condition-see [26, 16] for a discussion on Roe linearizations. This gives

$$
\hat{J}_{i+1 / 2}=J\left(\frac{1}{2}\left(Q_{i}+Q_{i+1}\right)\right) .
$$

\subsection{Source term discretization}

In this work, we calculate the source terms through weighted averages of the conserved variables, proportional to the wave strengths as

$$
\bar{S}_{i+1 / 2}=S\left(\frac{\lambda^{1}}{\left|\lambda^{1}\right|+\left|\lambda^{2}\right|} Q_{i+1}+\frac{\lambda^{2}}{\left|\lambda^{1}\right|+\left|\lambda^{2}\right|} Q_{i}\right) .
$$


In this work, we use an arithmetic average for model parameters such as hydraulic conductivity $K$, but other averages such as the harmonic average is also possible. This might be desirable, for example, if the difference in $K$ between two cells is large.

\subsection{Boundary conditions}

We use ghost cells to impose boundary conditions. We define either water depth or discharge in the ghost cell and compute the other boundary value through finite differencing.

Depth boundary condition If we impose the mass, $m_{f, *}$, in the ghost cell-and thus, $h_{f, *}$ is known-the unit discharge in the ghost cell, $q_{f, *}$, is calculated through Darcy's law as

$$
q_{f, *}=\frac{1}{2}\left(h_{i}+h_{f, *}\right) K_{i} \frac{\eta_{i}-\eta_{f, *}}{\Delta x},
$$

where the subscript $i$ denotes values in the inner cell.

Inflow boundary condition Similarly, if $q_{f, *}$ is imposed, the depth can be computed through Darcy's law as

$$
h_{f, *}=\sqrt{q_{f, i}^{2}-2 q_{f, *} \Delta x K_{i}^{-1}} .
$$

Species concentration boundary condition If we impose the species concentration $c_{*}$, we can make use of the fact that $\lim _{\tau \rightarrow 0} \psi_{c}=\partial_{c} c$ to compute the auxilliary variable, $\psi_{c, *}$, in the ghost cell through a forward difference as

$$
\psi_{c, *}=\frac{c_{i}-c_{*}}{\Delta x} .
$$

\subsection{Comparison between parabolic and hyperbolic time step size}

In this section, we only discuss the time step size for the hBE. The discussion of the time step size for the hADR is similar, but involves advective terms, which may be limiting the time step size more significantly than the diffusion terms. In such advection-dominated cases, the hyperbolization does not influence the time step size.

The efficiency of the hBE given in Sys. (4) can be assessed by comparing its eligible time step size with the parabolic equation's time step size, which can be constrained by a von Neumann stability analysis. Under the assumption of close to linear behavior from Eq. (1), the eligible time step size can be estimated such as in [4, 12] as

$$
\Delta t_{p} \leq S_{y} \frac{\Delta x^{2}}{4 K h}
$$


This assumption is valid for small gradients of the potential $h$. Due to the linearization, this constraint might be more restrictive than actually required. Nevertheless, it can be used as a first estimation. The eligible time step size of Sys. (4) is given by the classical Courant-Friedrichs-Lewy (CFL) condition as

$$
\Delta t_{h} \leq \Delta x \sqrt{\frac{S_{y} \tau}{K h}} .
$$

The hyperbolic equation is more efficient than the parabolic one in terms of time step size if

$$
\frac{\Delta t_{h}}{\Delta t_{p}}>1
$$

which gives a lower bound for the relaxation parameter $\tau$ as

$$
\tau>\frac{\Delta x^{2}}{16} \sqrt{\frac{S_{y}}{K h}},
$$

Thus, if values of $\tau$ that satisfy Eq. (29) are still small enough to ensure that the hyperbolic system in Eq. (4) converges to the parabolic Eq. (1) in each time step, using the hyperbolic form is more advantageous.

\section{Model verification}

In the following, we present a complete set of steady state and transient benchmarks to verify the numerical scheme for both the hBE and hADR. In test cases without analytical solution, we obtain the reference solution numerically using the ReacTran package [27]—an R language package for numerically solving general ADR. In all test cases involving the BE, the relaxation time was set to $\tau=0.2 \Delta x$, which satisfies Eq. (29). In test cases involving the $\mathrm{ADR}$, we use the same $\tau$ as for the cases involving the $\mathrm{BE}$, but here, the advection terms determine the time step size. $L_{\infty}$-norm errors for all test cases are summarized in Tab. 3 in App. B. Further, results from a mesh convergence study are reported in Tab. 4 in App. B.

\subsection{Subsurface flow test cases}

\subsubsection{Steady state test cases}

We run 2 simulations until they converge to a steady state, in order to verify that the steady states are correctly reproduced without spurious oscillations. The computational domain for all cases is $L=10 \mathrm{~m}$ long. At the left boundary, a constant discharge of $q(x=0)=1 \mathrm{~m}^{2} / \mathrm{s}$ is imposed. The downstream boundary condition imposes a fixed fluid depth equal to the analytical solution at this location. The CFL number for all simulations is set to 0.9. Tab. 1 summarizes the relevant simulation parameters for all 


\begin{tabular}{lcccc} 
Case & $S_{y}(x)(-)$ & $K(x)(\mathrm{m} / \mathrm{s})$ & $z(x)(\mathrm{m})$ & $\tau$ \\
\hline BE1 & 1 & $\sin (2 \pi x)+1.1$ & 0 & 0.004 \\
BE2 & 1 & $\operatorname{sgn}(\sin (1.2 \pi x))+1.1$ & 0 & 0.004 \\
BE3 & 1 & $11-x$ & $f(x)^{\dagger}$ & 0.004 \\
BE4 & $0.1 \sin (1.2 \pi x)+1$ & $11-x$ & $f(x)^{\dagger}$ & 0.004 \\
BE5 & $1-0.4 \exp \left(-2(x-5)^{2}\right)$ & $10-5 \exp \left(-2(x-5)^{2}\right)$ & 0 & 0.001 \\
\hline & ${ }^{\dagger} f(x)=\left\{\begin{array}{lll}\exp \left(-2(x-4)^{2}\right) & x<7 \\
1.5 & x \in[7,8] & \\
0 & \text { else }\end{array}\right.$
\end{tabular}

Table 1: Test cases for hBE: Summary of relevant simulation parameters

steady state test cases. For each test case, model predictions are compared against an analytical solution-see App. A for the derivation.

Fig. 2 shows the results for the water depth $h$ for each of these simulations. Model predictions using 500 cells are plotted with circles. The continuous line shows the reference solution. We omit a plot of the unit discharge $q$ for these test cases, as $q$ converges to the analytical solution of constant $1 \mathrm{~m}^{2} / \mathrm{s}$ up to machine accuracy in each test case-we compute an $L_{\infty}$-norm of about $10^{-14} \mathrm{~m}^{2} / \mathrm{s}$, see Tab. 3. The results prove that the numerical scheme preserves the discharge in moving equilibria up to machine accuracy in the presence of spatially varying conductivity and specific yield values. As seen in test cases BE3 and BE4, sharp discontinuities in the bed elevation $z$ are handled well.

\subsubsection{Transient test case}

We consider an initial value problem for the $\mathrm{hBE}$ in a domain with a length of $L=10 \mathrm{~m}$. At initial time, the fluid mass is constant $m_{f}(t=0)=3.0 \mathrm{~m}$. Relevant simulation parameters are given in Tab. 1, row BE5. We discretize the domain with 2000 cells. Fig. 3.a) shows model predictions and reference solutions at different time steps. The transient case is accurately reproduced by the augmented scheme.

Using the same test case, we study the model's sensitivity to the relaxation time $\tau$, which is varied from $10^{-5}$ to 0.1 . Fig. 3. $(\mathrm{b})$ shows model predictions and the reference solution for $t=0.03 \mathrm{~s}$. Large values of $\tau$, in this specific case in the order of magnitude between 0.01 and 0.1 , result in large deviations from the reference solution. As $\tau$ is decreased below 0.001, the model predictions converge to the reference solution.

As the value $\tau$ affects the time step size, see Eq. (27), it is desirable to choose $\tau$ as large as possible. Similar to the choice of mesh resolution, this results in a trade-off between efficiency and accuracy. Fig. 3(c) plots the $L_{\infty}$-normed error against the value of $\tau$. The dashed line marks the value where the parabolic time step is reached, see Eq. (26). Values of $\tau$ below 


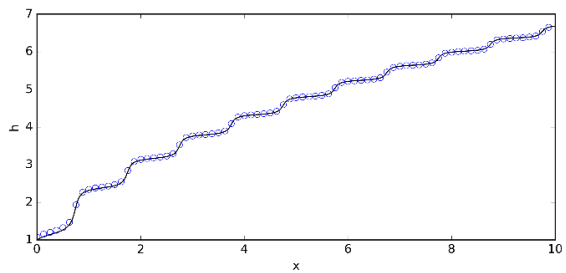

(a) BE1

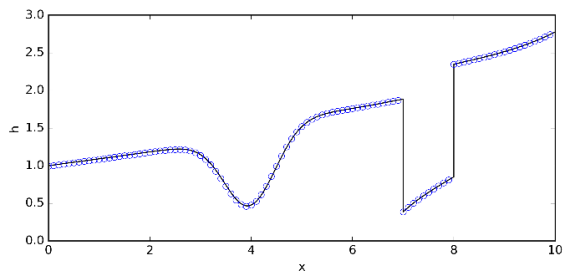

(c) BE3

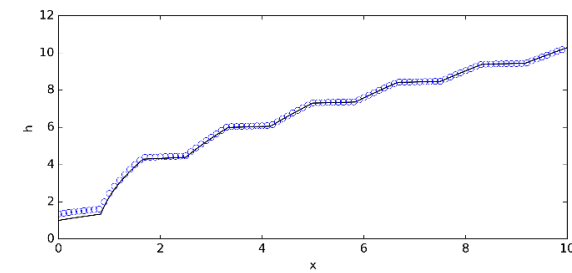

(b) BE2

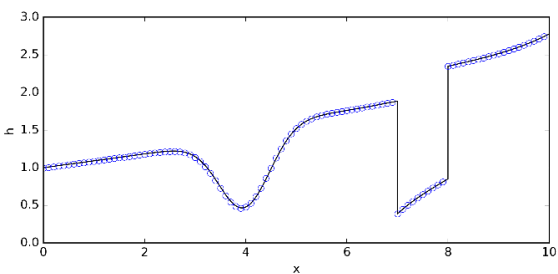

(d) BE4

Figure 2: Steady state test cases for hBE: Model prediction (circles) and analytical solution (continuous line) for $h$.

this line result in less efficient time steps compared with the original $\mathrm{BE}$. Consequently, values of $\tau$ above this line ensure the $\mathrm{hBE}$ is more efficient than the BE. In our specific case, no significant improvement is gained after $\tau=10^{-4}$.

\subsection{Reactive transport test cases}

\subsubsection{Steady state test cases}

We run two simulation runs until they reach a steady state. The domain is $L=25 \mathrm{~m}$ long. A constant species concentration of $c(x=0)=1$ is injected at the boundary at $x=0$. The concentration is reduced by a nonlinear reaction term along the domain. We discretize the domain with 500 cells. The constant injection at the boundary ensures that the system converges toward a steady state. We give the relevant simulation parameters in Tab. 2. rows ADR1 and ADR2. Fig. 4 shows that our scheme completely reproduces the reference solutions for $c$ at the steady state. Both test cases yield very similar results, because the advection dominates in both cases-the Peclet number is $P e \gg 1$ in both cases.

\subsubsection{Transient test cases}

We run transient simulation cases in a domain with length $L=100 \mathrm{~m}$, discretized with 1000 cells. We use periodic boundary conditions. Thus, simu- 


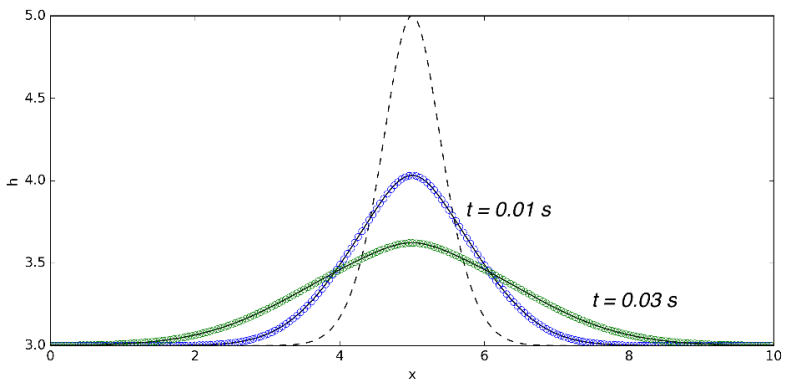

(a) BE5: Model prediction (circles) and reference solution (continuous line) for $\mathrm{h}$. Initial condition is shown with a dashed line.

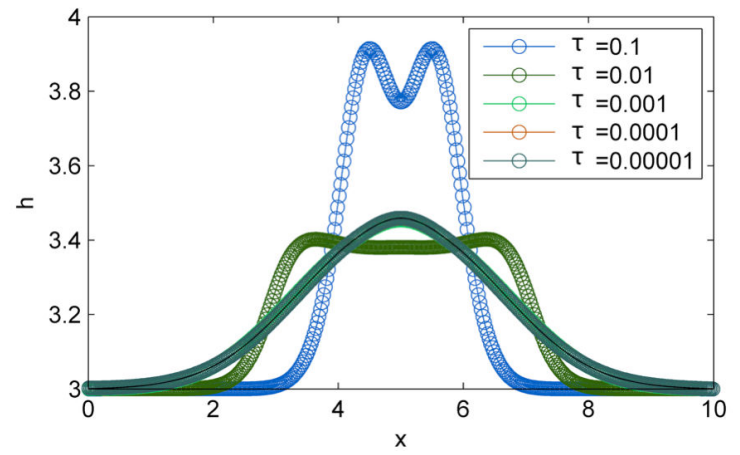

(b) BE5: Model predictions for different values of $\tau$ (circles) and reference solution (continuous line) at $t=0.03 \mathrm{~s}$.

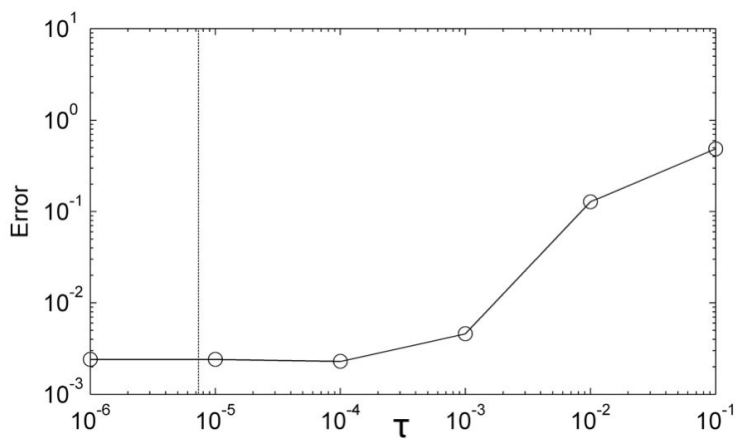

(c) $L_{\infty}$-normed errors plotted against $\tau$ (circles). The dashed line marks the value of $\tau$ that corresponds to the parabolic time step.

Figure 3: Transient test case for hBE: Model prediction (circles) and analytical solution (continuous line) for $h$. Initial condition in dashed line. 


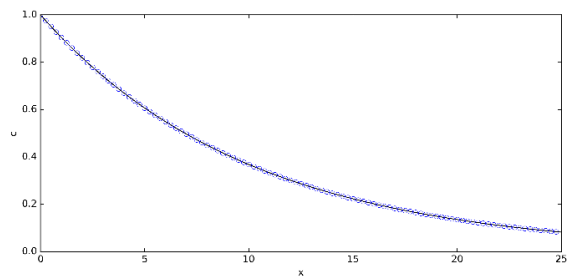

(a) ADR1

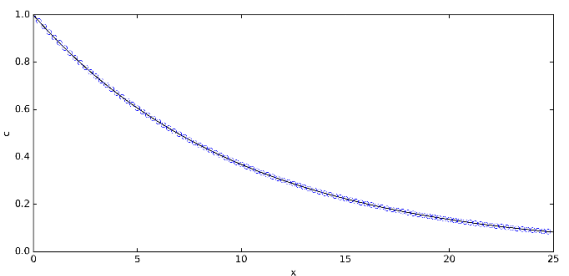

(b) ADR2

Figure 4: Steady state test case for hADR: Model prediction (circles) and analytical solution (continuous line) for $c$.

\begin{tabular}{lcccc} 
Case & $D(x)(-)$ & $v_{f}(x)(\mathrm{m} / \mathrm{s})$ & $R(c, x)(1 / \mathrm{s})$ & $\tau$ \\
\hline ADR1 & 0 & 1.0 & $0.1 c$ & 0.01 \\
ADR2 & $10^{-2}$ & 1.0 & $0.1 c$ & 0.01 \\
ADR3 & 0 & 1.5 & 0 & 0.02 \\
ADR4 & $10^{-3}$ & 0.0 & 0 & 0.02 \\
ADR5 & $10^{-2}$ & 0.5 & 0 & 0.02 \\
ADR6 & $10^{-2}$ & 0.1 & $10^{-3}$ & 0.02 \\
\hline
\end{tabular}

Table 2: Test cases for hADR: Summary of relevant simulation parameters

lation results only depend on the initial conditions, which are summarized in Tab. 2 for different simulation runs. In this table, ADR3 is a pure advection case, $\mathrm{ADR} 4$ is a pure diffusion case, $\mathrm{ADR} 5$ is an advection-reaction case, and finally, ADR6 is a full advection-diffusion-reaction case. For these cases, the analytical solution can be obtained as discussed in App. A. In all cases, the analytical solution (continuous line) is captured satisfactorilysee Fig. 5 .

\section{Modeling intrameander hyporheic flow and transport}

We present a simulation of hyporheic flow and transport through a $62 \mathrm{~m}$ long transect across a meander of the East River, Colorado, see Fig. 6(a). This transect has been studied in [9] to quantify geochemical exports from the hyporheic zone to the river. River water with high oxygen and nitrate concentrations enters the transect on the western boundary. The oxygen and nitrate are consumed in the hyporheic zone due to the presence of organic carbon sources, and are exported back to the river on the eastern boundary. Fig. 6(a) also shows the location of four measurement points where we compare our model predictions. The surface elevation is based on 


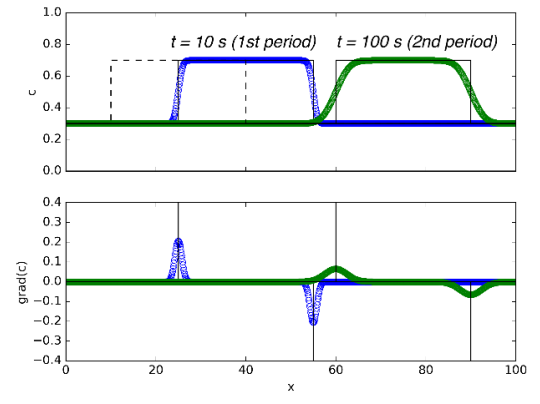

(a) ADR3

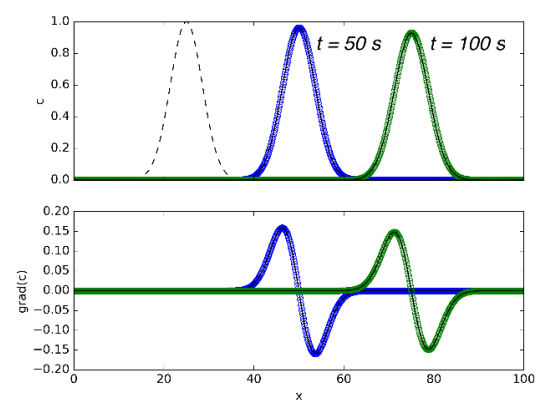

(c) ADR5

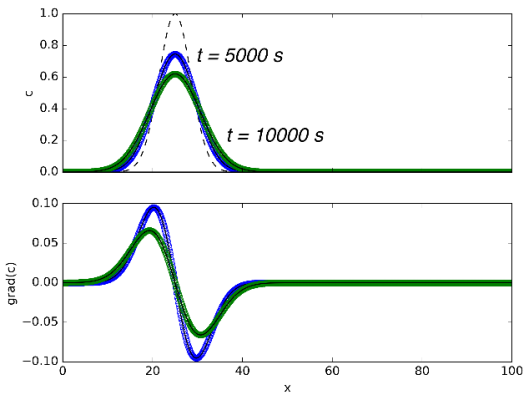

(b) $\mathrm{ADR} 4$

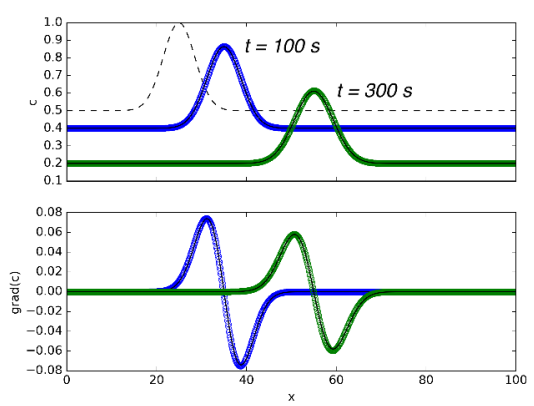

(d) ADR6

Figure 5: Transient test cases for hADR: Model prediction (circles) and analytical solution (continuous line) for $c$ and $\psi_{c}(\operatorname{grad}(c))$. 
a digital elevation model with $0.5 \mathrm{~m}$ resolution [33].

The geology of the transect consists of three distinct layers [9]. The top layer is a $0.5 \mathrm{~m}$-deep soil layer with low permeability, followed by a highly permeable $1 \mathrm{~m}$-deep gravel layer. The third layer, which makes up the majority of the domain, is a low permeability weathered shale layer. Our depth-averaged model is unable to resolve vertical heterogeneity in permeability. Hence, we assume that the permeable subsurface consists of weathered shale and that the impermeable boundary is $12.25 \mathrm{~m}$ below the surface-see Fig. 6.b). Based on field investigations [29], the conductivity of the weathered shale is set to $K=1.1 \times 10^{-5} \mathrm{~m} / \mathrm{s}$. The transect is discretized with 124 cells with a resolution of $0.5 \mathrm{~m}$, which corresponds to the data resolution. Using finer grids does not significantly improve the model predictions.

We obtain the initial conditions by running the model toward a steady state, using annually averaged constant head boundary conditions on both sides. Fig. 6(c) shows the time series that we averaged to get the head boundary conditions. The difference in the piezometric head creates a hydraulic gradient, which drives the flow through the subsurface transect. Fig. 6. d) shows the initial conditions we obtained. Our model predicts an average groundwater velocity of about $v_{f}=2.3 \times 10^{-7} \mathrm{~m} / \mathrm{s}$, which is an order of magnitude slower than the minimum velocity of $v_{f}=5 \times 10^{-6} \mathrm{~m} / \mathrm{s}$ predicted in [9]. The difference in model predictions is because our model is based on simplified physics and geology, while the model in [9] solves the three-dimensional Richards equation in a domain with several distinct vertical geological layers.

We apply the time series shown in Fig. 6(c) as head boundary conditions on both sides of the domain and simulate the transport of a dissolved electron acceptor (for example, oxygen or nitrate) through the intrameander hyporheic zone. We use a diffusion coefficient of $D=10^{-3}$, chosen rather high to qualitatively account for mechanical dispersion. We assume the electron acceptor to be consumed at the rate $R=\mathscr{C} c_{O}$, where $\mathscr{C}$ is a first-order reaction rate coefficient and $c_{O}$ is the dissolved concentration. The reaction rate coefficient accounts for the combined effect of the microbial community composition, the amount of organic carbon and other components, and the mineral composition in the soil-see, for example, [9, 15, 21]. In this study, we choose $\mathscr{C}=10^{-5} 1 / \mathrm{s}$ as it results in complete consumption of the electron acceptor within the meander, which is consistent with observations in [9]. We assume an initial concentration of $c_{O}(t=0)=0$ in the entire domain and use a fixed concentration of $c_{O}(x=0)=0.25$ at the upstream (western) boundary.

Fig. 7 shows the model predictions for piezometric head against field measurements. The agreement is fair, the overall dynamics of the measured piezometric head are captured by the model. Overall, the agreement gets worse as the flow propagates through the domain. This is most likely due to numerical diffusion and an incomplete representation of the physics. The agreement at MCP1 is the best, because its location is close to the 


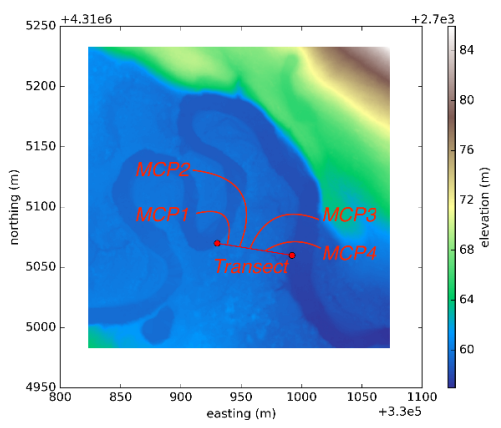

(a) Location of the transect and measurement points

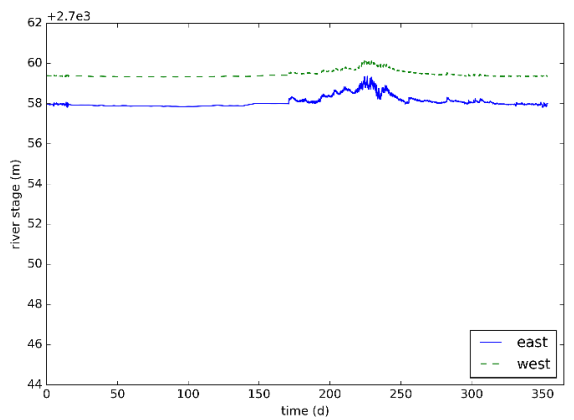

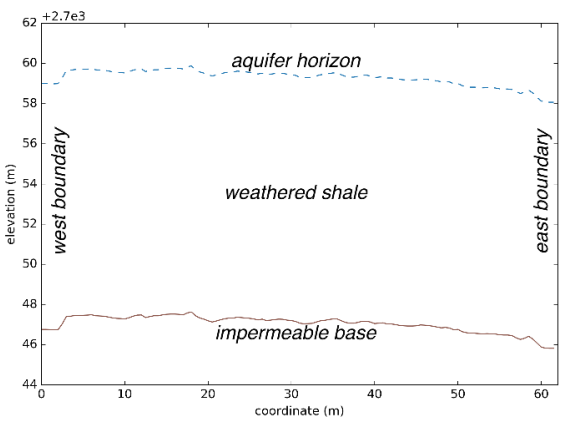

(b) Geology of the transect with impermeable bed (continuous line) and surface elevation (dashed line)

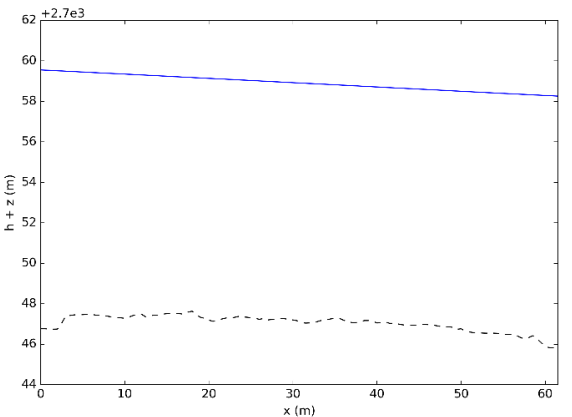

(c) Boundary conditions at the eastern (d) Initial conditions for piezometric (continuous line) and western bound- head (continuous line) and impermeable aries (dashed line) base (dashed line)

Figure 6: Intrameander hyporheic flow and transport: Study site and boundary and initial conditions. 

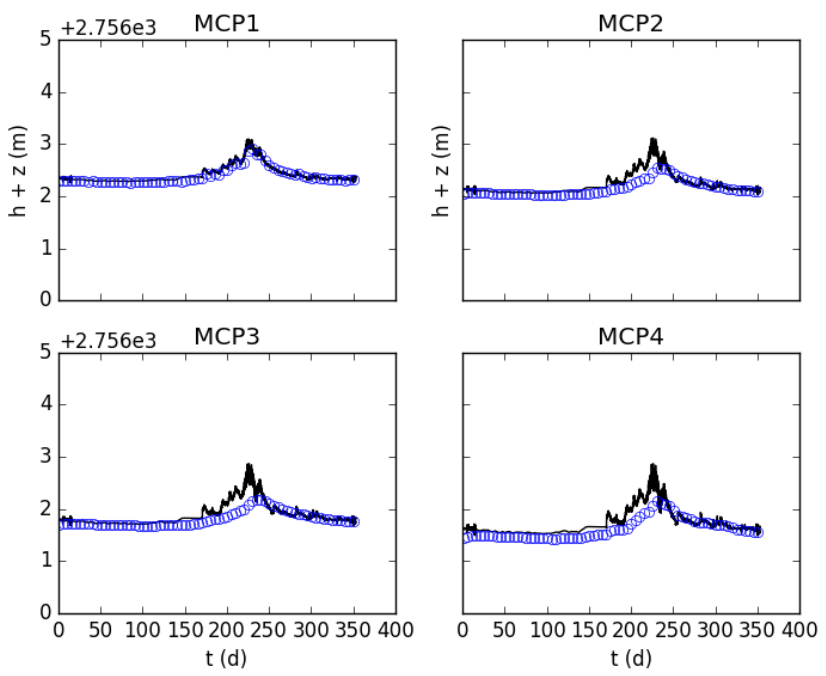

Figure 7: Intrameander hyporheic flow and transport: Model predictions (circles) and field measurements (continuous line) for piezometric head at 4 points along the transect.

boundary and is therefore less affected by numerical diffusion. At the other locations, the predicted curve lags behind the measurement and does not reproduce the peak accurately. High-frequency fluctuations are smoothed out by the model prediction. This may be a consequence of the simplified geology. Our model considers the entire domain to be shale. In reality, the top of the domain consists of a more permeable gravel layer, which may be responsible for the higher frequencies observed in the measurement data. While model predictions might be further improved by modifying the conductivity to account for the top layers, the current predictions are sufficiently accurate for our purpose of presenting a proof of concept modeling study.

Fig. 8 shows model predictions for the electron acceptor concentration along the transect at different times. The electron acceptor concentration reaches a quasi-steady state at about 10 days, when the consumption balances out with the fluxes. The dynamic observed in the piezometric head in Fig. 7 does not significantly disturb this equilibrium, because the diffusive fluxes outweigh the advective fluxes. While the model predictions qualitatively resemble the model predictions in [9], our hADR model with its simple reaction term is not comparable to the biogeochemical reaction network model utilized therein. 


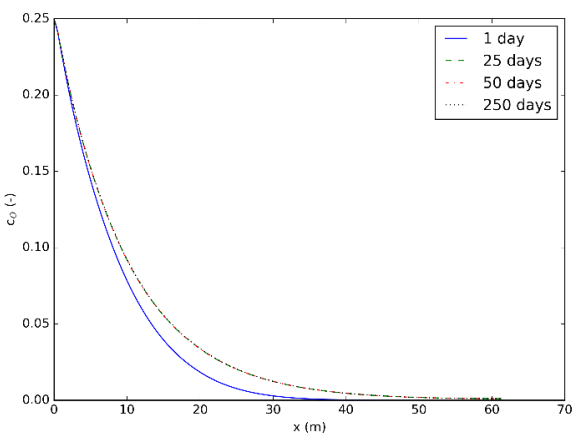

Figure 8: Intrameander hyporheic flow and transport: Model predictions for dissolved electron acceptor concentration along the transect at different times.

\section{Conclusions}

We applied Cattaneo's relaxation approach [3] to the Boussinesq groundwater flow equation and the diffusive terms of the advection-diffusion-reaction equation. This transforms both equations into first order hyperbolic systems with source terms. We used the augmented solver approach [10, 20], combined with a geometric reinterpretation of the source terms, to solve the hyperbolic system of partial differential equations.

Transient and steady state benchmark test cases show that the relaxed hyperbolic system is equivalent to the original parabolic equations. The unit discharge in the $\mathrm{BE}$ appears as a primary variable in the hyperbolized equations. The numerical scheme enables us to compute the steady state unit discharge with machine accuracy. These properties are desirable for coupled reactive transport simulations, because the advective flux in the $\mathrm{ADR}$ is determined by the unit discharge in the BE.

The case study of intrameander groundwater flow and reactive transport shows that the model is capable of modeling real world cases to some extent. The depth-averaged groundwater flow model is unable to reproduce the fast component of the subsurface flow, but the overall dynamics are accurately modeled. The groundwater flow model can be extended to higher dimensions to overcome the limitations of depth-averaged modeling. The coupled ADR computes feasible results but suffers from an even more simplified representation of the processes. The case study serves as a proof of concept, and the reaction terms can be computed in a more sophisticated manner if needed. Adding more species to the model for multicomponent reactive transport simulations is straight-forward, but has not been explored in this work.

Overall, using Cattaneo's relaxation approach to hyperbolize parabolic 
governing equations and using an augmented solver to numerically solve the resulting hyperbolic system is shown to be a viable approach. The presented method can be applied to other areas of hydrological modeling that are governed by parabolic equations. However, additional studies are necessary to completely assess the advantages and limitations of this method for hydrological applications.

\section{Acknowledgments}

This work is supported as part of the Watershed Function Scientific Focus Area funded by the U.S. Department of Energy, Office of Science, Office of Biological and Environmental Research under Award Number DE-AC0205CH11231. This work is supported as part of the Interoperable Design of Extreme-scale Application Software (IDEAS) by the U.S. Department of Energy, Office of Science, Office of Biological and Environmental Research, Award Number DE-AC02-05CH11231. Adrián Navas-Montilla is partially funded through Gobierno de Aragón through the Fondo Social Europeo. The data of the case study is based upon work supported as part of the Sustainable Systems Scientific Focus Area, funded by the U.S. Department of Energy, Office of Science, Office of Biological and Environmental Research under Award Number DE-AC02-05CH11231.

\section{Author disclosure statement}

No competing financial interests exist.

\section{A Derivation of analytical solutions}

\section{A.1 Subsurface flow}

\section{A.1.1 Steady state solutions}

Imposing a constant discharge $q_{0}$ in Eq. (1) gives the ordinary differential equation

$$
\frac{d \eta}{d x}=\frac{q_{0}}{K(x)(\eta(x)-z(x))},
$$

which can be solved for $m_{f}=(\eta-z) S_{y}$.

\section{A.2 Reactive transport}

\section{A.2.1 Transient solutions}

Using constant flow velocity, $v_{f}$, and diffusion coefficient, $D$, and imposing

$$
c(x, 0)=\exp \left(-C_{1}\left(x-C_{2}\right)^{2}\right)
$$




\begin{tabular}{lccc} 
Case & Time & $L_{\infty}(\mathrm{m})$ & $L_{\infty}(q)$ \\
\hline BE1 & steady & $3.9 \times 10^{-2}$ & $3.3 \times 10^{-14}$ \\
BE2 & steady & $3.5 \times 10^{-1}$ & $3.3 \times 10^{-14}$ \\
BE3 & steady & $3.6 \times 10^{-2}$ & $2.5 \times 10^{-14}$ \\
BE4 & steady & $3.6 \times 10^{-2}$ & $2.5 \times 10^{-14}$ \\
BE5 & $0.03 \mathrm{~s}$ & $1.2 \times 10^{-3}$ & - \\
ADR1 & steady & $4.2 \times 10^{-5}$ & - \\
ADR2 & steady & $1.6 \times 10^{-5}$ & - \\
ADR3 & $100 \mathrm{~s}$ & $1.4 \times 10^{-1}$ & - \\
ADR4 & $10,000 \mathrm{~s}$ & $3.3 \times 10^{-5}$ & - \\
ADR5 & $100 \mathrm{~s}$ & $9.5 \times 10^{-3}$ & - \\
ADR6 & $300 \mathrm{~s}$ & $6.3 \times 10^{-3}$ & -
\end{tabular}

Table 3: $L_{\infty}$-norm errors for the verification test cases. Errors in $q$ are only computed for steady BE cases.

\begin{tabular}{lcc} 
Cell size $(\mathrm{m})$ & $L_{\infty}(\mathrm{m})$ & $L_{\infty}(q)$ \\
\hline 0.5 & $5.7 \cdot 10^{0}$ & $3.8 \cdot 10^{-14}$ \\
0.05 & $7.7 \cdot 10^{-1}$ & $6.5 \cdot 10^{-14}$ \\
0.02 & $3.5 \cdot 10^{-1}$ & $3.3 \cdot 10^{-14}$
\end{tabular}

Table 4: $L_{\infty}$-norm errors for the verification case BE2 for different grid sizes.

as an initial condition in Eq. (1) allows to extend the analytical solution given in [5] as

$$
c(x, t)=\frac{1}{\sqrt{1+4 C_{1} D t}} \exp \left(\frac{-C_{1}\left(x-C_{2}-v_{f} t\right)^{2}}{1+4 C_{1} D t}\right)-t R(c(x, t)),
$$

where $C_{1}$ and $C_{2}$ are free parameters. Eq. (32) can be solved for $c$ exactly if $R(c(x, t))$ is constant.

\section{B Errors for the verification test cases}

We give the $L_{\infty}$-norm errors for each verification test case in Tab. 3 . We carried out simulations on coarser meshes and observed that in all cases the model prediction converges toward the reference solution as the mesh is refined with $\mathcal{O}(h)$. We provide $L_{\infty}$-norm errors for the test case BE2 in Tab. 4, which evidences the first order convergence rate of the scheme. 


\section{References}

[1] J. Bear. Dynamics of Fluids in Porous Media. Elsevier Publishing, New York, USA, 1972.

[2] U. Boyraz and C. M. Kazezyllmaz-Alhan. Solutions for groundwater flow with sloping stream boundary: analytical, numerical and experimental models. Hydrology Research, 49:1120-1130, 2018.

[3] C. Cattaneo. A form of heat-conduction equations which eliminates the paradox of instantaneous propagation. Comptes Rendus Mathématique, 247:431-433, 1958.

[4] D. Caviedes-Voullième, P. García-Navarro, and J. Murillo. Verification, conservation, stability and efficiency of a finite volume method for the 1D Richards equation. Journal of Hydrology, 480, 2013.

[5] C. Cebollada-Gimeno. Estudio y simulación de flujos turbulentos bidimensionales con aplicación a canales abiertos con regiones de separación de flujo. Master's thesis, Universidad de Zaragoza, Zaragoza, Spain, 2018.

[6] F. Clarens and C. A. Peters. Mitigating climate change at the carbon water nexus: A call to action for the environmental engineering community. Environmental Engineering Science, 33:719-724, 2016.

[7] R. Courant, K. Friedrichs, and H. Lewy. Über die partiellen Differenzengleichungen der mathematischen Physik. Mathematische Annalen, 100:32-74, 1928.

[8] G. Dal Maso, P. G. LeFloch, and F. Murat. Definition and weak stability of nonconservative products. Journal de Mathématiques Pures et Appliquées, 74:483-548, 1995.

[9] D. Dwivedi, C. I. Steefel, B. Arora, M. Newcomer, J. D. Moulton, B. Dafflon, B. Faybishenko, P. Fox, P. Nico, N. Spycher, R. Carroll, and K. H. Williams. Geochemical exports to river from the intrameander hyporheic zone under transient hydrologic conditions: East River mountainous watershed, Colorado. Water Resources Research, 54:84568477, 2018.

[10] L. Gosse. A well-balanced scheme using non-conservative products designed for hyperbolic systems of conservation laws with source terms. Mathematical Models and Methods in Applied Sciences, 11:339-365, 2001.

[11] F. He and F. Roe. A novel numerical scheme based on active flux method for the hyperbolic heat equations in multidimensional space. In AIAA Aviation 2019 Forum, Dallas, Texas, 2019. 
[12] N. M. Hunter, M. S. Horritt, P. D. Bates, M. D. Wilson, and M. G. F. Werner. An adaptive time step solution for raster-based storage cell modelling of floodplain inundation. Advances in Water Resources, 28:975-991, 2005.

[13] S. Jin, L. Pareschi, and G. Toscani. Diffusive relaxation schemes for multiscale discrete-velocity kinetic equations. SIAM Journal on $\mathrm{Nu}$ merical Analysis, 35:2405-2439, 1998.

[14] S. Jin and Z. Xin. The relaxation schemes for systems of conservation laws in arbitrary space dimensions. Communications on Pure and Applied Mathematics, 38:235-277, 1995.

[15] S. Krause, C. Tecklenburg, M. Munz, and E. Naden. Streambed nitrogen cycling beyond the hyporheic zone: Flow controls on horizontal patterns and depth distribution of nitrate and dissolved oxygen in the upwelling groundwater of a lowland river. Journal of Geophysical Research: Biogeosciences, 118:54-67, 2013.

[16] R. J. LeVeque. Finite Volume Methods for Hyperbolic Problems. Cambridge University Press, Cambridge, UK, 2002.

[17] R. J. LeVeque. A well-balanced path-integral f-wave method for hyperbolic problems with source terms. Journal of Scientic Computing, 48:209-226, 2011.

[18] L. Li, K. Maher, A. Navarre-Sitchler, J. Druhan, C. Meile, C. Lawrence, J. Moore, J. Perdrial, P. Sullivan, A. Thompson, L. Jin, E. W. Bolton, S. L. Brantley, W. E. Dietrich, K. U. Mayer, C. I. Steefel, A. Valocchi, J. Zachara, B. Kocar, J. McIntosh, B. M. Tutolo, M. Kumar, E. Sonnenthal, C. Bao, and J. Beisman. Expanding the role of reactive transport models in critical zone processes. Earth-Science Reviews, 165:280-301, 2017.

[19] A. Mazaheri and H. Nishikawa. First-order hyperbolic system method for time-dependent advection-diffusion problems. Technical Report NASA-TM-2014-218175, National Institute of Aerospace Associates, Hampton, VA, USA, 2014.

[20] J. Murillo and A. Navas-Montilla. A comprehensive explanation and exercise of the source terms in hyperbolic systems using Roe type solutions. Application to the 1D-2D shallow water equations. Advances in Water Resources, 98:70-96, 2016.

[21] R.-C. Naranjo, R.-G. Niswonger, and C.-J. Davis. Mixing effects on nitrogen and oxygen concentrations and the relationship to mean residence time in a hyporheic zone of a riffle-pool sequence. Water Resources Research, 51:7202-7217, 2015. 
[22] H. Nishikawa. A first-order system approach for diffusion equation. I: Second-order residual-distribution schemes. Journal of Computational Physics, 227:315-352, 2007.

[23] H. Nishikawa. Beyond interface gradient: A general principle for constructing diffusion schemes. In 40th Fluid Dynamics Conference and Exhibit, Chicago, Illinois, 2010.

[24] H. Nishikawa. New-generation hyperbolic Navier-Stokes schemes: $O(1 / h)$ speed-up and accurate viscous/heat fluxes. In 20th AIAA Computational Fluid Dynamics Conference, Honolulu, Hawai'i, 2011.

[25] C. Parés. Numerical methods for nonconservative hyperbolic systems: A theoretical framework. SIAM Journal on Numerical Analysis, 44:300-321, 2006.

[26] P. L. Roe. Approximate Riemann solvers, parameter vectors, and difference schemes. Journal of Computational Physics, 43:357-372, 1981.

[27] K. Soetaert and F. Meysman. R-package ReacTran: Reactive transport modelling in R. Technical report, Nederlands Instituut voor Zeeonderzoek, Yerseke, the Netherlands, 2019.

[28] C. I. Steefel, C. A. J. Appelo, B. Arora, D. Jacques, T. Kalbacher, O. Kolditz, V. Lagneau, P. C. Lichtner, K. U. Mayer, J. C. L. Meeussen, S. Molins, D. Moulton, H. Shao, J. Šimøunek, N. Spycher, S. B. Yabusaki, and G. T. Yeh. Reactive transport codes for subsurface environmental simulation. Computational Geosciences, 19:445-478, 2015.

[29] T. Tokunaga, J. Wan, K. H. Williams, W. Brown, A. Henderson, Y. Kim, A. P. Tran, M. E. Conrad, M. Bill, R. W. H. Carroll, W. Dong, Z. Xu, A. Lavy, B. Gilbert, S. Romero, J. N. Christensen, B. Faybishenko, B. Arora, E. R. Siirila-Woodburn, R. Versteeg, J. Raberg, J. E. Peterson, and S. S. Hubbard. Depth- and time-resolved distributions of snowmelt-driven hillslope-subsurface flow and transport and their contributions to surface waters. Water Resources Research, 55:94749499, 2019.

[30] E. F. Toro and G. I. Montecinos. Advection-diffusion-reaction equations: Hyperbolization and high-order ADER discretizations. SIAM Journal on Scientific Computing, 36:A2423-A2457, 2014.

[31] I. Toumi. A weak formulation of Roe's approximate Riemann solver. Journal of Computational Physics, 102:360-373, 1992.

[32] D. Vanzo, A. Siviglia, and E. F. Toro. Pollutant transport by shallow water equations on unstructured meshes: Hyperbolization of the model and numerical solution via a novel flux splitting scheme. Journal of Computational Physics, 321:1-20, 2016. 
[33] H. M. Wainwright and H. K. Williams. LiDAR collection in August 2015 over the East River Watershed, Colorado, USA. https://doi. org/10.21952/WTR/1412542, 2017. 